\title{
Efeito agudo do alongamento estático na força muscular de mulheres idosas
}

\author{
Acute effect of static stretching on muscle force in older women
}

\author{
André Luiz Demantova Gurjão \\ Nelson Hilário Carneiro ${ }^{2}$ \\ Raquel Gonçalves \\ Rodrigo Ferreira de Moura ${ }^{1}$ \\ Sebastião Gobbi
}

1 Universidade Estadual Paulista. Instituo de Biociências. Laboratório de Atividade Física e Envelhecimento. Rio Claro,SP. Brasil

2 Universidade do Oeste Paulista. Presidente Prudente, SP. Brasil.

Recebido em 05/01/09 Revisado em 20/03/09 Aprovado em 02/12/09
Resumo - O objetivo deste estudo foi investigar, em mulheres idosas, o efeito agudo do alongamento estático sobre a taxa de desenvolvimento de força pico (TDFP) e contração voluntária máxima (CVM). A amostra foi composta por 10 mulheres idosas (idade 68,5 $\pm 7,0$ anos; peso 70,9 $\pm 8,1 \mathrm{~kg}$; estatura 159,4 $\pm 6,0 \mathrm{~cm}$; índice de massa corporal 28,0 $\pm 3,8 \mathrm{~kg} / \mathrm{m}^{2}$ ). A TDFP e a CVM foram testadas no exercício Leg Press, antes e após as condições controle ou alongamento (três séries de 30 segundos de alongamento estático do quadríceps femoral), em dois dias diferentes (24 horas de intervalo). A TDFP foi determinada como a inclinação mais íngreme da curva, calculada dentro da janela regular de $20 \mathrm{~ms}(\Delta \mathrm{Força/ \Delta Tempo})$, para os primeiros 200 ms após o início da força muscular. A CVM foi determinada como o maior ponto registrado na tentativa. Em cada dia, apenas uma condição foi testada e a ordem de emprego para cada condição foi determinada aleatoriamente. A intensidade do alongamento foi determinada pelo limiar de dor muscular. Quatro avaliações pós-condições (pós-tratamento; 10; 20 e 30 minutos) foram realizadas para acompanhar o comportamento da força muscular. A ANCOVA 2x5, seguida do teste post-hoc de Scheffé, não mostrou interações, condição vs. momento, significativas $(P>0,05)$ para a TDFP e CVM. Em conclusão, séries agudas de alongamento estático para o quadríceps femoral não afetam a capacidade de produzir força muscular rapidamente e máxima de mulheres idosas.

Palavras-chave: Envelhecimento; Aquecimento; Curva força-tempo Isométrica.

Abstract - The objective of this study was to investigate the acute effect of static stretching on the peak rate of force development (PRFD) and maximum voluntary contraction (MVC) in older women. Ten women $(68.5 \pm 7.0$ years; $70.9 \pm 8.1 \mathrm{~kg} ; 159.4 \pm 6.0 \mathrm{~cm}$; body mass index: $\left.28.0 \pm 3.8 \mathrm{~kg} / \mathrm{m}^{2}\right)$ were studied. MVC and PRFD were determined by leg press exercise before and after the control or stretching condition (three sets of 30 seconds of static stretching of the quadriceps) on two different days (interval of 24 hours). PRFD was determined as the steepest slope of the curve, calculated within regular windows of 20 milliseconds ( $\Delta$ force/ $\Delta$ time) for the first 200 milliseconds after the onset of contraction. MVC was determined as the highest value recorded in each set. Only one condition was tested on each day and the order of application of each condition was determined randomly. The stretching intensity was evaluated by the muscle pain threshold. Four post-condition assessments (post-treatment, 10, 20, and 30 minutes) were performed to monitor muscle strength. ANCOVA 2x5, followed by the Scheffé post-hoc test, showed no significant interactions between conditions vs. times $(P>0.05)$ for PRFD or MVC. In conclusion, acute bouts of static stretching of the quadriceps femoris do not affect the ability of rapid and maximum muscle force production in older women.

Key words: Aging; Warn-up; Isometric force-time curve. 


\section{INTRODUÇÃO}

Durante o processo de envelhecimento, alterações estruturais e funcionais no sistema neuromuscular levam a significativos declínios nas expressões da força muscular e flexibilidade, ambos componentes da capacidade funcional ${ }^{1,2}$. $\mathrm{O}$ decréscimo da funcionalidade desse sistema pode comprometer de maneira parcial ou completa a realização das atividades da vida diária, acarretando em maior dependência e redução da qualidade de vida de adultos idosos ${ }^{3}$. Este fato é particularmente relevante para mulheres, as quais apresentam maior expectativa de vida do que homens, reduzida reserva funcional e maior declínio da força muscular de membros inferiores ${ }^{4,5}$.

Neste sentido, a prática regular de exercícios físicos com objetivo de melhorar o desempenho da força muscular e flexibilidade tem sido recomendada como meio de atenuar ou reverter os efeitos deletérios relacionados ao envelhecimento e/ou fatores a ele associados sobre esses componentes da capacidade funcional ${ }^{6}$. Contudo, a melhor maneira de incluir o desenvolvimento de ambos os componentes em uma única sessão de treinamento não está clara ${ }^{7}$, especialmente em idosos.

Exercícios de alongamento têm sido tradicionalmente incorporados no início das sessões de treinamento com o objetivo de reduzir o risco de lesões ${ }^{8}$, melhorar o desempenho ${ }^{9}$ e aumentar a amplitude de movimento de uma articulaçãa ${ }^{10}$. Contudo, Pope et al. ${ }^{11}$ têm concluído que exercícios de alongamento não diminuem o risco de ocorrência de lesões. Em adição, resultados contraditórios têm sido reportados a respeito do efeito agudo do alongamento sobre a função neuromuscular.

Recentes trabalhos não têm demonstrado qualquer efeito do exercício de alongamento estático sobre o desempenho da força muscular ${ }^{12,13}$. Em contrapartida, vários autores têm confirmado que diferentes rotinas de alongamento podem levar à redução aguda, tanto da força muscular isométrica quanto dinâmica ${ }^{14,15}$, por um período de tempo tão longo quanto 120 minutos $^{16}$. Power et al. ${ }^{16}$, por exemplo, observaram decréscimos na contração voluntária máxima por um período de tempo 12 vezes superior ao reportado por Behm et al. ${ }^{17}$, mesmo empregando um volume de alongamento 4,4 vezes inferior (270 vs. 1200 segundos, respectivamente). Assim, fica evidente que a relação dose-resposta entre o volume de alongamento empregado e o decréscimo na produção de força muscular e seu padrão temporal de comportamento ainda perma- nece incerto, indicando a necessidade de maiores investigações.

Em adição, alguns estudos que demonstraram reduções agudas na força muscular empregaram em seus protocolos experimentais, rotinas de alongamento não representativas àquelas realizadas em situações práticas ou recomendadas por organizações voltadas à saúde quando se objetiva melhora da flexibilidade ${ }^{17,18}$.

Para adultos idosos, atuais recomendações internacionais incluem rotinas de alongamento estático compostas por 3-4 séries com duração de 10-30 segundos ${ }^{6}$. Levando este fato em consideração, não tem sido estabelecido se períodos curtos de alongamento estático podem levar ao decréscimo agudo na produção de diferentes expressões da força muscular em adultos idosos e a possível duração desse comportamento. Assim, o objetivo deste estudo foi analisar, em mulheres idosas, o efeito agudo de exercícios de alongamento estático sobre o comportamento da taxa de desenvolvimento de força pico e contração voluntária máxima.

\section{PROCEDIMENTOS METODOLÓGICOS}

Participaram do estudo 10 mulheres idosas (idade $68,5 \pm 7,0$ anos; peso $70,9 \pm 8,1 \mathrm{~kg}$; estatura 159,4 $\pm 6,0 \mathrm{~cm} ;$ IMC $28,0 \pm 3,8 \mathrm{~kg} / \mathrm{m}^{2}$ ) fisicamente ativas. As participantes atendiam regularmente um programa de exercício físico, com frequência de três vezes por semana, a pelo menos três meses. Nenhuma lesão músculo-esquelética recente foi relatada. As participantes foram informadas verbalmente sobre os procedimentos a que seriam submetidas e assinaram um Termo de Consentimento Livre e Esclarecido aprovado pelo Comitê de Ética em Pesquisa da Universidade Estadual Paulista.

\section{Delineamento Experimental}

Cada participante visitou o laboratório por quatro ocasiões diferentes, com 24 horas de intervalo. Nas duas primeiras visitas, foram realizadas as mensurações antropométricas e familiarização aos procedimentos da rotina de alongamento e avaliação da Cf-t isométrica de membros inferiores. Nas duas visitas subsequentes, a Cf-t isométrica foi registrada adotando o seguinte protocolo experimental: registro de três Cf-t isométricas referentes ao momento pré-tratamento, com intervalo de recuperação de três minutos entre os registros. Após intervalo de 5 minutos, as participantes realizaram uma de duas condições experimentais: controle (sem alongamento) ou alongamento. As avaliações da 
Cf-t isométrica, após o emprego dessas condições, foram realizadas imediatamente após a condição adotada (pós-tratamento) e durante um período de tempo de 30 minutos relativos ao final de cada condição (10; 20; e 30 minutos), com o objetivo de examinar o padrão temporal de possíveis alterações. A ordem de emprego para cada condição (controle ou alongamento) foi determinada pelo método de aleatorização do tipo cross-over balanceado. Para a condição controle, as participantes permaneceram repousadas em decúbito ventral sobre uma maca, por um período de tempo similar à duração total da rotina de alongamento $(\sim 2,5 \mathrm{~min})$. A Cf-t isométrica que apresentou a maior contração voluntária máxima (CVM), no período pré-tratamento, foi selecionada para as comparações com as Cf-t isométricas obtidas nos momentos pós-tratamento; 10; 20 e 30 minutos. As participantes foram orientadas a não realizar atividades físicas intensas durante o período de avaliações. Todos os procedimentos foram realizados na mesma hora do dia para minimizar possíveis variações circadianas no comportamento da força muscular.

\section{Mensuração da curva força-tempo}

isométrica (Cf-t isométrica)

O esforço isométrico máximo de extensão unilateral de joelhos e quadris (Leg Press) foi avaliado por meio de um transdutor de força (modelo 5000 $\mathrm{N}^{\mathrm{TM}}$, EMG System do Brasil ${ }^{\circledR}$, São José dos Campos, SP, Brasil), com as participantes posicionadas sobre o assento do equipamento, similar ao descrito por Sahaly et al..$^{19} \mathrm{e}$ com os joelhos flexionados a 90 graus. No momento pré-avaliação, as avaliadas foram instruídas a realizar suas contrações voluntárias máximas (CVM) "tão rápido quanto possível", por cinco segundos. Tão logo iniciado o esforço, as participantes foram encorajadas verbalmente a realizarem seus esforços máximos.

A aquisição do sinal proveniente do transdutor de força foi realizada por meio de um amplificador de sinais analógicos (modelo EMG 800C-USB, EMG System do Brasil ${ }^{\circledR}$, São José dos Campos, SP, Brasil), com frequência de amostragem de $2000 \mathrm{~Hz}$. O sinal obtido pelo amplificador foi armazenado em disco rígido e analisado posteriormente off-line. Como primeiro procedimento, o sinal bruto do transdutor de força foi digitalmente filtrado por um filtro passa-baixa Butterworth de segunda-ordem, zero-lag e frequência de corte de $15 \mathrm{~Hz}$. O início da produção de força muscular foi definido como o ponto no qual o valor de força muscular excedeu 7,5 $\mathrm{N}$ acima da linha de base ${ }^{20}$. A CVM foi determina- da como o mais alto valor registrado durante toda a avaliação. A taxa de desenvolvimento de força pico (TDFP) foi determinada como a inclinação mais íngreme da Cf-t isométrica ( $\triangle$ Força/ $\Delta$ Tempo), calculada dentro de janelas regulares de $20 \mathrm{~ms}$, para os primeiros $200 \mathrm{~ms}$ a partir do início da produção de força muscular20.

\section{Protocolo de alongamento estático}

A rotina de alongamento empregada no presente estudo foi elaborada, levando em consideração as atuais recomendações internacionais para adultos idosos ${ }^{6}$. Foram realizadas três séries de alongamento estático passivo para o quadríceps femoral das participantes, com 30 segundos de duração. $\mathrm{O}$ intervalo de recuperação entre cada série foi de 30 segundos.

A rotina de alongamento adotada na sessão experimental foi realizada sobre uma maca com as participantes posicionadas em decúbito ventral. $\mathrm{O}$ joelho do membro dominante de cada participante foi lentamente flexionado pelo pesquisador na tentativa de levar os calcanhares em direção ao glúteo. $\mathrm{O}$ limite do alongamento foi determinado como a amplitude de movimento articular atingida no momento em que a participante relatou o início da sensação de dor. É importante ressaltar que antes das sessões experimentais, todas as participantes foram familiarizadas aos procedimentos adotados para a realização da rotina de alongamento estático. Durante a sessão de familiarização, as participantes foram instruídas a identificar e relatar ao pesquisador o início da sensação de dor durante a flexão do joelho do membro dominante.

\section{Tratamento estatístico}

Inicialmente, os dados foram tratados a partir de procedimentos descritivos (média \pm erro padrão). Para verificação do princípio da esfericidade, utilizou-se o teste de Mauchly. A análise de covariância (ANCOVA) $2 \times 5$ foi empregada para verificar possíveis diferenças nos valores de TDFP e CVM nas comparações entre condições (controle e alongamento) e momentos (pré-tratamento; póstratamento; 10; 20 e $30 \mathrm{~min}$ ), apresentando como co-variável a idade das participantes. $\mathrm{O}$ teste posthoc de Scheffé foi empregado para a identificação das diferenças específicas nas variáveis em que os valores de $\mathrm{F}$ encontrados foram superiores ao critério estabelecido de significância estatística $(\mathrm{P}<$ $0,05)$. Todas as informações foram processadas no pacote computacional Statistica ${ }^{\mathrm{TM}}$, versão 5.0. 


\section{RESULTADOS}

A figura 1 apresenta o comportamento da TDFP (A) e CVM (B) antes e após as condições controle e alongamento. Nenhum efeito principal de condição ou interação condição vs. momento foram observados para a TDFP $(\mathrm{P}=0,92$ e 0,99; respectivamente) e CVM ( $\mathrm{P}=0,87$ e 0,91; respectivamente). Assim, não foi verificado nenhum efeito que possa ser atribuído à rotina de alongamento estático empregada. Embora o efeito principal de tempo tenha sido verificado para a TDFP $(\mathrm{P}=0,02)$ e CVM $(\mathrm{P}<0,01)$, diferenças significativas em relação aos valores pré-tratamento foram observadas apenas para a CVM obtida no décimo minuto para a condição experimental.

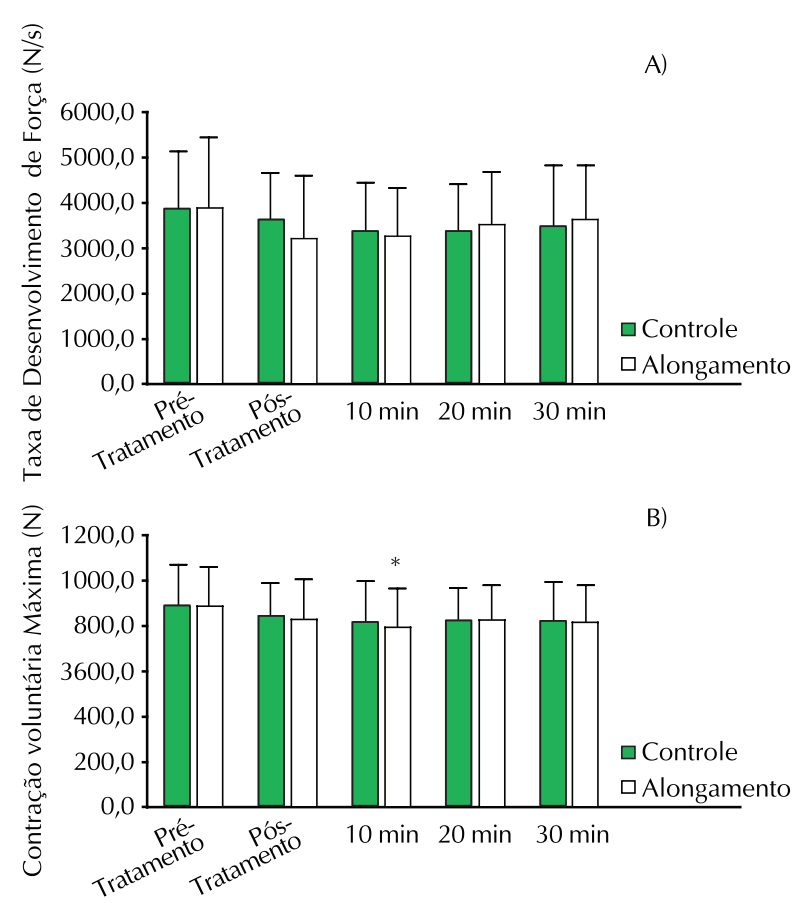

Figura 1. Comportamento da taxa de desenvolvimento de força pico (A) e contração voluntária máxima (B) antes (pré-tratamento) e após (pós-tratamento; 10; 20 e 30 minutos) as condições controle e experimental (alongamento estático). Valores em média \pm desvio-padrão $(n=10)$ * Diferença significativa $(P<$ $0,05)$ em relação aos valores pré-tratamento.

\section{DISCUSSÃO}

O achado principal desse estudo foi que a rotina de alongamento empregada não afetou negativamente a capacidade de mulheres idosas em produzir força muscular rapidamente ou máxima (figuras 1-A e 1-B, respectivamente). A falta de modificações significativas no comportamento da força muscular, após o emprego de rotinas de alongamento, está de acordo com outros resultados encontrados na literatura ${ }^{12,13,21,22}$. Em contrapartida, uma variedade de autores tem demonstrado reduções transitórias no desempenho neuromuscular, fenômeno comumente conhecido como déficit de força muscular induzido por alongamento ${ }^{14-17}$.

Embora o déficit de força muscular induzido por alongamento deva ser considerado durante a prescrição de programas de exercícios físicos, é importante salientar a existência que uma variedade de fatores que podem determinar seu comportamento. Dentre estes fatores, o tempo total de alongamento é uma importante variável a ser considerada.

O tempo de duração do alongamento, associado ao número de séries, determina o volume da rotina de alongamento empregada. Muitos estudos que procuraram investigar o comportamento da força muscular, após a realização de exercícios de alongamento, têm utilizado em seus delineamentos protocolos com volumes demasiadamente altos. Fowles et al..$^{23}$, por exemplo, observaram redução na CVM dos flexores plantares ao realizarem $30 \mathrm{~min}$ de alongamento estático (13 séries de $135 \mathrm{~s}$ cada) para os flexores plantares. Ao considerar a grande variabilidade nos volumes dos protocolos de alongamento encontrados na literatura, torna-se difícil realizar comparações com outros estudos.

Embora não esteja claro o comportamento da relação dose-resposta entre o tempo total de alongamento e o déficit de força muscular, diferentes estudos têm apontado que maiores volumes de alongamento parecem ser mais eficazes em gerar decréscimos na força muscular, em comparação aos menores volumes ${ }^{15,24}$. Siatras et al..$^{15}$ observaram que 30 segundos de alongamento estático reduziu o pico de torque isométrico de sujeitos jovens em 8,5\%. Esse decréscimo foi inferior em comparação aos $16 \%$ de redução verificada após 60 segundos de alongamento estático.

Ryan et al. ${ }^{22}$ procuraram investigar o efeito agudo de diferentes volumes de alongamentos estáticos passivos (2; 4 e 8 minutos) no desempenho da TDFP e CVM dos flexores plantares. Decréscimos na TDFP atribuíveis às rotinas de alongamento foram observadas somente após o emprego das rotinas com volumes de 4 e 8 minutos. Para CVM, nenhuma alteração induzida pelos diferentes volumes de alongamento foi verificada. Nosso estudo adotou as recomendações internacionais atuais para adultos idosos quanto ao volume de alongamento, empregando três séries com 30 segundos de duração ${ }^{6}$. Esse protocolo não levou à redução na TDFP e CVM de mulheres idosas.

Bazzet-Jones et al. ${ }^{25}$ também não observaram alterações na taxa de desenvolvimento de força 
e CVM obtidas em exercício multiarticular, após realização de uma rotina de alongamento, com volume similar ao adotado em nosso estudo (três séries de 30 segundos por grupo muscular).

McBride et al. ${ }^{26}$ demonstraram que as alterações mediadas pelo alongamento sobre o comportamento de diferentes parâmetros da Cf-t isométrica, em adultos jovens, podem ser influenciadas pelo tipo de exercício. Os autores procuraram investigar o efeito do alongamento estático sobre o quadríceps (três séries de 33 s), durante a produção de força isométrica em exercícios multi e monoarticular. Redução significativa na TDF, para os primeiros $400 \mathrm{~ms}$, foi observada durante exercício multiarticular, sem alteração na CVM. Contudo, para o exercício monoarticular, apenas a CVM foi reduzida. Os autores sugerem que a diferença no comportamento da Cf-t isométrica entre os exercícios mono e multiarticular pode ter ocorrido devido a um mecanismo compensatório da musculatura sinergista. Assim, mesmo que o alongamento tenha gerado alterações no desempenho da musculatura agonista, o desempenho de força muscular no exercício multiarticular pode não ser alterado, em decorrência da ação compensatória de outros grupos musculares não alongados. De qualquer forma, essa é uma hipótese que necessita ser testada em adultos idosos.

O padrão temporal de modificação na força muscular mediado pelo alongamento não tem sido investigado sistematicamente. A relação entre o volume de alongamento empregado e o tempo de duração do déficit de força muscular é bastante controversa. Na presente investigação, tanto a TDFP quanto a CVM obtidas após o emprego da rotina de alongamento apresentaram comportamento similar ao da condição controle, em qualquer período de tempo analisado (pós-tratamento; 10; 20; e 30 minutos). Embora diferença significativa tenha sido verificada para a CVM no décimo minuto na condição alongamento, não foi observada interação condição vs. momento significativa. Power et al. ${ }^{16}$ observaram decréscimos na CVM por um período de 120 minutos, após a realização de 270 segundos de alongamento estático, em sujeitos jovens. Em contra partida, Behm et al. ${ }^{17}$, também em sujeitos jovens, verificaram reduções na CVM por apenas 10 minutos, após empregarem uma rotina bastante prolongada de alongamento estático (1200 segundos). Levando em consideração o fato de não estar claro a relação dose-resposta sobre o padrão temporal do déficit de força muscular mediado pelo alongamento estático, é possível que protocolos realizados com volumes superiores ao adotado em nosso trabalho possam alterar tanto a magnitude quanto o padrão temporal dessas respostas em mulheres idosas.

Embora o presente estudo apresente achados relevantes, não se pode desprezar o possível efeito da quantidade de grupos musculares alongados no comportamento da força muscular. Em nosso delineamento experimental, apenas o quadríceps femoral foi submetido ao protocolo de alongamento. Ao considerar que o desempenho de força em exercícios multiarticulares também depende da ação de outros grupos musculares, o emprego de exercícios de alongamento em mais de um grupo muscular pode levar a resultados diferentes dos observados. Gurjão et al. ${ }^{27}$, por exemplo, verificaram reduções significativas para a TDFP e CVM de mulheres idosas, quando mais de um grupo muscular foi alongado.

Apesar da presente investigação não ter avaliado os possíveis mecanismos associados ao déficit de força muscular mediado pelo alongamento estático, dois mecanismos primários são propostos na tentativa de explicar tal fenômeno: a) alterações na unidade músculo-tendão (incremento da complacência) e b) alterações neurais (excitabilidade inibida dos neurônios motores alfa ou do sistema nervoso central) $)^{24}$.

O comportamento da TDFP pode ser influenciado por uma série de fatores, dentre eles, ambos os mecanismos anteriormente citados. Wilson et al. ${ }^{28}$ reportaram significativa relação entre a complacência da unidade músculo-tendão e o desempenho de força muscular isométrica. Os autores sugerem que uma unidade músculo-tendão mais rígida é mais eficiente durante a fase inicial de transmissão de força, incrementando, desse modo, a expressão da TDFP. Edman e Tsuchiya ${ }^{29}$ concluíram que durante exercícios de alongamento a complacência da unidade músculo-tendão é aumentada, ou seja, apresenta redução em sua rigidez. Assim, o possível aumento da complacência da unidade músculo-tendão, após a rotina de alongamento, aumentaria o tempo necessário para o alongamento dessa estrutura, durante a produção de força muscular. Isso significa que a capacidade dessa estrutura em transmitir rapidamente a força muscular gerada pela musculatura para o osso está diminuída, especialmente, nos momentos iniciais de produção de força ${ }^{30}$. Isso afetaria diretamente o comportamento da TDFP. Em nosso estudo, o fato da TDFP não ter sido reduzida significativamente, após a realização do protocolo de alongamento estático, sugere que alterações na unidade músculo-tendão 
podem não ter ocorrido.

Ainda que modificações na ativação muscular não possam ser descartadas como fator contribuinte para as respostas observadas, é possível que esse mecanismo apresente menor participação em comparação às alterações na unidade músculotendão. Conforme pontuado por Fowles et al. ${ }^{23}$, uma possível inibição autogênica na ativação muscular mediada pelos órgãos tendinosos de Golgi necessitariam de uma rotina de alongamento extremamente intensa e prolongada para ser ativada. Nossa rotina de alongamento estático foi realizada no limiar de dor e com curto período de duração. Ainda segundo Fowles et al. ${ }^{23}$, os receptores de dor também podem reduzir a ativação neural da musculatura. Porém, percepções de desconforto ou dor não estão presentes durante a avaliação da Cf-t isométrica, no período pós-alongamento. Esse fato sugere que reduções na ativação muscular mediadas por esta via podem não ter ocorrido.

\section{CONCLUSÃO}

A análise dos resultados mostra que, em mulheres idosas, a realização de séries de alongamento estático para o quadríceps femoral não afeta a capacidade de produzir força muscular rapidamente e máxima em exercícios multiarticulares. Ainda que nossos resultados não mostrem prejuízos para a TDFP e CVM, deve-se salientar que apenas a produção de força isométrica foi avaliada e uma melhor compreensão desse fenômeno em relação a outras expressões da força muscular pode apresentar relevantes implicações nas recomendações para o treinamento de ambos os componentes da capacidade funcional (força muscular e flexibilidade) em adultos idosos.

\section{Agradecimentos}

Os autores agradecem a FUNDUNESP, PROEX, FAPESP e CAPES.

\section{REFERÊNCIAS BIBLIOGRÁFICAS}

1. Akima H, Kano Y, Enomoto Y, Ishizu M, Okada M, Oishi Y, Katsuta S, Kuno S. Muscle function in 164 men and women aged 20-84 yr. Med Sci Sports Exerc 2001;33(2):220-226.

2. Holland GJ, Tanaka K, Shigematsu R, Nakagaichi M. Flexibility and physical functions of older adults: A Review. J Aging Phys Act 2002;10(2):169-206.

3. Warburton DER, Gledhill N, Quinney A. The effects of changes in muscleskeletal fitness on health. Can J Appl Physiol 2001;26(2):161-216.

4. Kwon IS, Schrager M, Talbot LA, Fozard JL, Metter EJ. Relationship between muscle strength and the time taken to complete a standardized walk-turn-walk test. J Gerontol A Biol Sci Med Sci 2001;56(9):B398-B404.

5. Lynch NA, Metter EJ, Lindle RS, Fozard JL, Tobin JD, Roy TA, Fleg JL, Hurley BF. Muscle quality. I. Ageassociated differences between arm and leg muscle groups. J Appl Physiol 1999;86(1):188-194.

6. Nelson ME, Rejeski WJ, Blair SN, Duncan PW, Judge JO, King AC, Macera CA, Castaneda-Sceppa C, American College of Sports Medicine, American Heart Association. Physical activity and public health in older adults: recommendation from the American College of Sports Medicine and the American Heart Association. Circulation 2007;116(9):1094-1105.

7. Rubini EC, Costa ALL, Gomes PSC. The Effects of Stretching on Strength Performance. Sports Med 2007;37(3):213-224.

8. Knudson D. Stretching during warm-up: Do we have enough evidence? J Orthop Sports Phys Ther 1999;70(1):24-27.

9. Shrier I, Gossal K. Myths and truths of stretching: Individualized recommendations for healthy muscles. Physician Sports Med 2000;28(1):57-63.

10. Feland JB, Myrer JW, Schulthies SS, Fellingham GW, Measom GW. The effect of duration of stretching of the hamstring muscle group for increasing range of motion in people aged 65 years or older. Phys Ther 2001;81(5):1110-1117.

11. Pope RP, Herbert RD, Kirwan JD, Graham BJ. A randomized trial of pre-exercise stretching for prevention of lower-limb injury. Med Sci Sports Exerc 2000;32(2):271-277.

12. Alpkaya U, Koceja D. The effects of acute static stretching on reaction time and force. J Sports Med Phys Fitness 2007;47(2):147-150.

13. Cramer JT Housh TJ, Johnson GO, Weir JP, Beck TW, Coburn JW. An acute bout of static stretching does not affect maximal eccentric isokinetic peak torque, the joint angle at peak torque, mean power, electromyography, or mechanomyography. J Orthop Sports Phys Ther 2007;37(3):130-139.

14. Behm DG, Kibele A. Effects of differing intensities of static stretching on jump performance. Eur J Appl Physiol 2007;101(5): 587-594.

15. Siatras TA, Mittas VP, Mameletzi DN, Vamvakoudis EA. The duration of the inhibitory effects with static stretching on quadriceps peak torque production. J Strength Cond Res 2008;22(1):40-46.

16. Power K, Behm DG, Cahill F, Carroll M, Young W. An acute bout of static stretching: effects on force and jumping performance. Med Sci Sports Exerc 2004;36(8):1389-1396.

17. Behm DG, Button DC, Butt JC. Factors affecting force loss with prolonged stretching. Can J Appl Physiol 2001;26(3):261-272.

18. Avela J, Finni T, Liikavainio T, Niemelä E, Komi PV. Neural and mechanical responses of the triceps surae muscle group after $1 \mathrm{~h}$ of repeated fast passive stretches. J Appl Physiol 2004;96(6):2325-2332.

19. Sahaly, R, Vandewalle, H, Driss, T, and Monod, H. Maximal voluntary force and rate of force development in humans - importance of instruction. Eur J Appl Physiol 2001;85(3-4):345-350. 
20. Aagaard P, Simonsen EB, Andersen JL, Magnusson P, Dyhre-Poulsen, P. Increased rate of force development and neural drive of human skeletal muscle following resistance training. J Appl Physiol 2002;93(4):1318-1326.

21. Handrakis JP, Southard VN, Abreu JM, Aloisa M, Doyen MR, Echevarria LM, Hwang H, Samuels C, Venegas SA, Douris PC. Static Stretching Does Not Impair Performance in Active Middle-Aged Adults. J Strength Cond Res. 2009 Out 7. [Epub ahead of print].

22. Ryan ED, Beck TW, Herda TJ, Hull HR, Hartman MJ, Stout JR, Cramer JT. Do practical durations of stretching alter muscle strength? A dose-response study. Med Sci Sports Exerc 2008;40(8):1529-1537.

23. Fowles JR, Sale DG, MacDougall JD. Reduced strength after passive stretch of the human plantar flexors. J Appl Physiol 2000;89(3):1179-1188.

24. Ogura Y, Miyahara Y, Naito H, Katamoto S, Joki J. Duration of static stretching influences Muscle force production in hamstring Muscles. J Strength Cond Res 2007;21(3):788-792.

25. Bazett-Jones DM, Winchester JB, McBride JM. Effect of potentiation and stretching on maximal force, rate of force development, and range of motion. J Strength Cond Res 2005;19(2):421-426.

26. McBride JM, Deane R, Nimphius S. Effect of stretching on agonist-antagonist muscle activity and muscle force output during single and multiple joint isometric contractions. Scand J Med Sci Sports 2007;17(1): 54-60.
27. Gurjão ALD, Gonçalves R, de Moura RF, Gobbi S. Acute effect of static stretching on rate of force development and maximal voluntary contraction in older women. J Strength Cond Res 2009;23(7):2149-2154.

28. Wilson GJ, Murphy AJ, Pryor JF. Musculotendinous stiffness: Its relationship to eccentric, isometric, and concentric performance. J Appl Physiol 1994;76(6):2714-2719.

29. Edman KA, Tsuchiya T. Strain of passive elements during force enhancement by stretch in frog muscle fibres. J Physiol 1996;490(Pt 1):191-205.

30. Narici MV, Maganaris CN. Plasticity of the muscletendon complex with disuse and aging. Exerc Sport Sci Rev 2007;35(3):126-134.
Endereço para correspondência
Sebastião Gobbi.
Departamento de Educação Física
Avenida 24 A, 1515. Bairro: Bela Vista
CEP: 13506-900 - Rio Claro, SP. Brasil.
E-mail: sgobbi@rc.unesp.br 